\title{
Study on Selection Methods of Parents and Crossover in Genetic Algorithm
} doi : https://doi.org/10.32628/CSEIT217256

\section{Dr. K. Santhi ${ }^{1}$, Dr. V. Vinodhini ${ }^{2}$}

${ }^{1}$ Associate Professor, Department of Information Technology, Dr.N.G.P Arts and Science College, Coimbatore, Tamilnadu, India.

2Professor, Department of Information Technology, Dr.N.G.P Arts and Science College, Coimbatore, Tamilnadu, India

\section{ABSTRACT}

Article Info

Volume 7, Issue 2

Page Number: 280-285

Publication Issue :

March-April-2021

\section{Article History}

Accepted : 07 April 2021

Published : 13 April 2021
Genetic Algorithms are the population based search and optimization technique that mimic the process of Genetic and Natural Evolution. Genetic algorithms are very effective way of finding an Optimized solution to a complex problem. Performance of genetic algorithms mainly depends on various factors such as selection of efficient parents and type of genetic operators which involve crossover and mutation operators etc. This paper will help the people to acquire the knowledge about various strategies of selecting parents and description about standard crossover operators.

Keywords: Genetic Algorithm(GA), Initial Population, Fitness function, Selection of Parents, Crossover, Mutation

\section{INTRODUCTION}

In this current scenario, Genetic Algorithm (GA) plays a vital role in the field of computer science and operation research (GA). GA is a part of Evolutionary Computing, popular search optimization techniques based on the principles of Genetics and Natural Selection method. Genetic Algorithm was proposed by John Holland (Father of GA). It is used to get the optimized result for both the constrained and unconstrained problems. Some of the major application of GA's are engineering, medicine, bioinformatics, forecasting, manufacturing, agriculture etc.

Genetic Algorithm operations begins with set of solution (each solution is represented as chromosome) called population. Each gene in the chromosome represents the characteristics of the solution. Part of the population is selected for reproduction. Generally, the selection depends on the value of fitness function. Selected chromosomes is called parents. From the parents new and best chromosomes generated are called offspring's for the next generation.

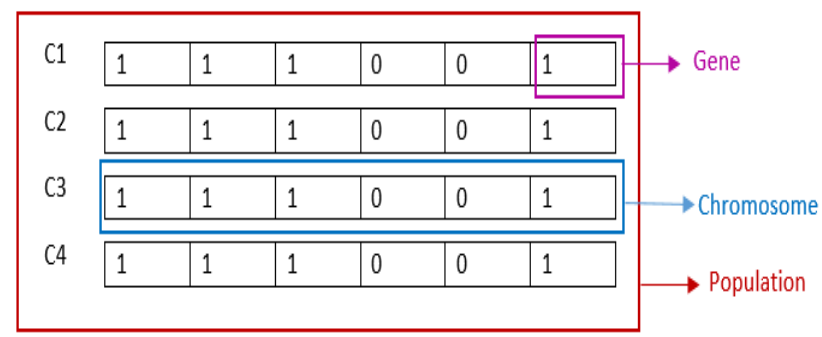

Figure 1. Representing Gene, Chromosome and Population 


\section{METHODS AND MATERIAL}

\section{Working of Genetic Algorithm}

Phases involved in the working of genetic algorithm are Initialisation, Fitness Function, Selection of parents, Crossover and Mutation

\subsection{Initialisation}

First step would be defining the population for the problem which contain number of individuals, each containing their own set of chromosomes.

\subsection{Fitness function}

This fitness function helps to evaluate the fitness score of each individuals in the population. Based on the fitness score the individuals will be selected for the reproduction.

\subsection{Selection}

In this selection phase, it selects the pairs of fittest individuals from the population and allows them to pass their genes/characteristics to the next generation. Individuals with maximum fitness have more chance to be selected rather than individuals with minimum fitness value. Various selection methods are available. They are

2.3.1 Random Selection method: In this selection technique, the parents are randomly selected from the given current population for the crossover operation using the uniform random number generator. This method is sometimes little disruptive when compared to other selection strategies[2].

2.3.2 Roulette Wheel Selection: It is also called as Fitness proportionate selection, is a genetic operator used in the genetic algorithm for selecting the parents for recombination. In this strategy, parents are selected from the given population based on the probability of its fitness value of each member. Probability can be calculated by dividing the fitness of each individual by the total fitness of the given set of population [2][1][4].

$$
\mathrm{P}(\mathrm{i})=\frac{f(i)}{\sum_{j=1}^{n} f(J)}
$$

Where $\mathrm{i}$ - denotes the individuals in the population, $\mathrm{n}$ denotes the total number of individuals in the population set. This implies that a chromosome which has the least value of cost will have the highest probability of being selected.

Let us consider an example

TABLE 1 : EXAMPLE FOR SELECTION OF PARENTS

\begin{tabular}{|l|l|l|}
\hline Candidate & $\begin{array}{l}\text { Fitness } \\
\text { value }\end{array}$ & $\begin{array}{l}\text { Percentage of } \\
\text { Total Fitness }\end{array}$ \\
\hline A & 109 & 28.09 \\
\hline B & 76 & 19.59 \\
\hline C & 50 & 12.89 \\
\hline D & 153 & 39.43 \\
\hline Total & 388 & 100 \\
\hline
\end{tabular}

First column represents the candidate, second column represents fitness value of the candidate and the third column value represents the probability of the candidate being selected as parents for recombination. For example, the candidate $\mathrm{D}$ has the probability of $39.43 \%(153 / 388)$ as parent when compared to other candidates in the population.

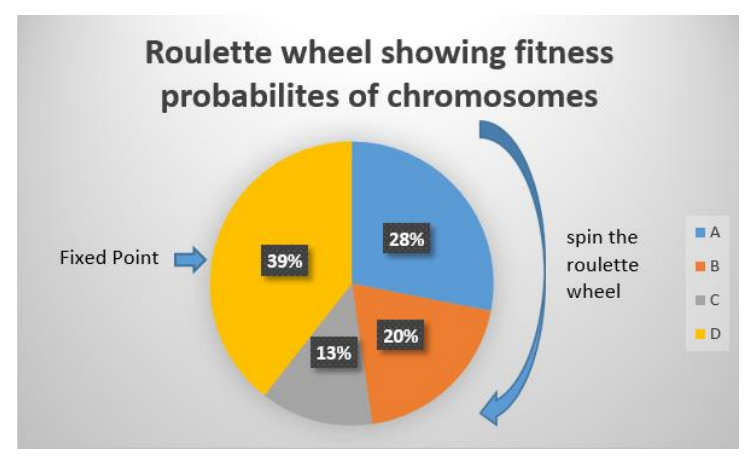

Figure 2. Roulette wheel selection

Advantage of this method is that the chromosome which has maximum fitness value has high chance of becoming a parent, because that chromosome occupies more space in the Roulette wheel. Most probably better chromosome will get the opportunity to become the parent. Drawback of this technique is that the chromosome with least probability value will have very few chances of becoming parent.[3] 
2.3.3 Stochastic Universal Sampling:This technique used in genetic algorithm for selecting parents from the population. This method was introduced by James Baker. It is similar to Roulette Wheel selection, in which it has only one fixed point where as in this method it has more than one (multiple) fixed points as shown in the figure. Points are equally spaced and number of pointers is equal to the individuals to be selected. All the parents are chosen in just one spin rotation of the wheel itself. This method is considered as fair because it gives selection chance to the weaker members of the population.[1]

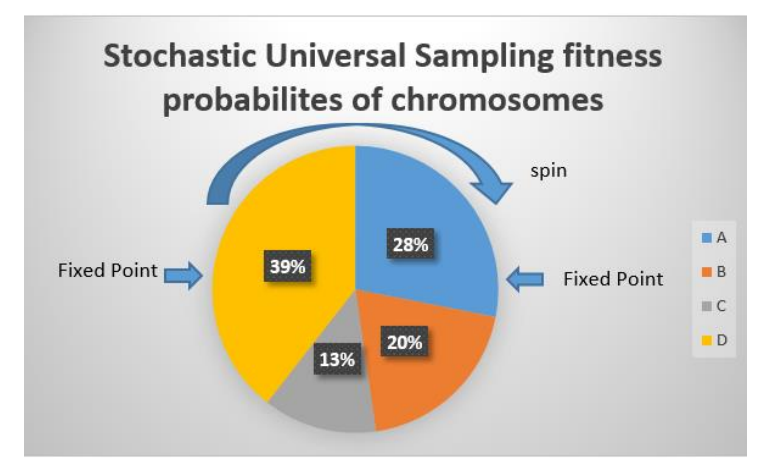

Figure 3. Stochastic Universal Sampling Fitness

For example, from the above figure consider the number of individuals to be selected as 2 . The distance between the pointer is $1 / 2$ and a randomly generated number in the range $[0,0.5]$ which gives the position of the pointer. The distance between the pointer is 0.5 . Two individuals will be selected for each spin.

2.3.4 Rank Selection: In this technique, every chromosome receives its rank by sorting the chromosome based on their fitness value. The chromosome with highest fitness value is ranked as $\mathrm{N}$ (Let $\mathrm{N}$ be the number of chromosome in the population) and the next highest value is ranked as $\mathrm{N}-1$, etc. The chromosome with the least fitness value will be given the rank as 1 . This ranking system leads to each individual having an almost equal share in the pie chart as shown in the figure. So each chromosome has approximately same probability of getting selected as parent.[1]
Situation before ranking (graph of fitness) Situation after ranking (graph of order numbers) .[3]. The selection of individual is given as [4]

$$
\mathrm{P}(\mathrm{i})=\frac{\operatorname{rank}(i)}{n X(n-1)} \quad-(2)
$$

This method lead to slower convergence, because the best chromosomes do not differ so much from others. Computationally expensive as a sorting is also required [5]

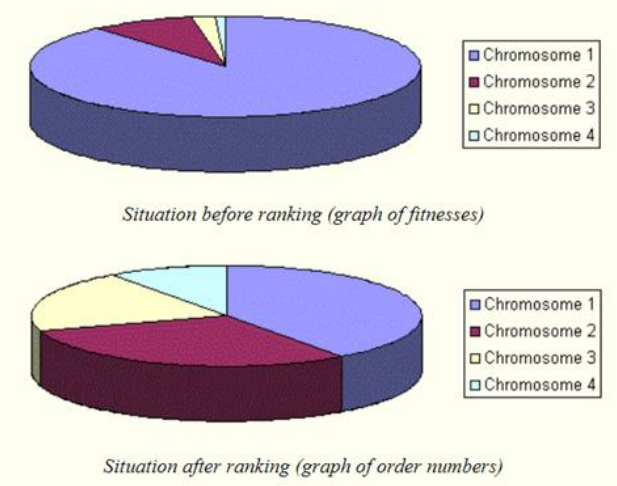

Figure 4. Rank Selection

\subsubsection{Tournament Selection:}

This selection strategy used for selecting the fittest chromosome from the current population. Selected candidates are then passed to the next generation. Steps involved in the tournament selection are (i) Select $\mathrm{k}$ individuals from the generation and perform a tournament amongst them (ii) From the $\mathrm{k}$ individuals select the best individual from them. The winner of the tournament is the chromosome with highest fitness. Repeat the above steps until the desired amount of population is reached. This method can be implemented efficiently since it doesn't require any sorting. It also preserves the diversity and provides the optimal solution. [1]

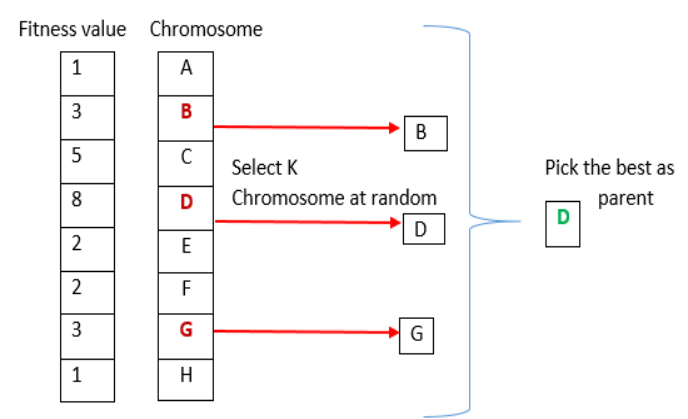

Figure 5. Tournament Selection 
2.3.6. Elitism selection: In this selection strategy only a limited number of individuals with the best fitness values are taken and pass to next generation, avoiding the crossover or mutation operators of individuals with good genetics.[5]

2.3.7. Truncation Selection: In this method chromosomes are sorted based on their fitness value. Only the best individuals are selected for the reproduction of new population. Parents are selected based on the threshold value. Threshold value helps to select the parents in the specified proportion $50 \%$ - $10 \%$ of its population. The chromosome which has values less than threshold value do not produce new population and they are discarded [1]

\subsection{Crossover}

This is the most significant part in the genetic algorithm. For mating, two individuals are selected from the population and a crossover point is chosen at random in order to generate superior offspring.

This section discusses about the various types of standard crossover operators. [8]

2.4.1 1-Point Crossover: It is a simple crossover technique used for random Genetic Algorithm applications. This method first selects two parents used for crossover and randomly selects a crossover point over a parents. Tails of the two parents are swapped in order to generate two new offspring

\begin{tabular}{|c|c|c|c|c|c|c|c|c|c|}
\hline Parent 1: & 1 & 2 & 3 & 4 & 5 & 6 & 7 & 8 & 9 \\
\hline Parent 2: & 1 & 3 & 5 & 7 & 9 & 2 & 4 & 6 & 8 \\
\hline Offspring 1: & 1 & 2 & 3 & 4 & 9 & 2 & 4 & 6 & 8 \\
\hline Offspring 2: & 1 & 3 & 5 & 7 & 5 & 6 & 7 & 8 & 9 \\
\hline
\end{tabular}

Figure 6. 1 Point Crossover

2.4.2 K-Point crossover: It is a simplest crossover technique where $\mathrm{k}$ takes the value from $1,2, \ldots . \mathrm{n}$. If $\mathrm{k}$ takes the value 1 then it is called 1-point crossover, $\mathrm{k}=2$ then it is called 2-point crossover and so on. It selects two parents from the population for crossover and selects randomly $\mathrm{k}$ crossover points. Pair of offspring's are created by combining the parents at crossover point.
A simple example is shown below with $\mathrm{K}=2$ i.e., two random points are chosen on the chromosomes where the genetic material is exchanged at these points to generate new offspring's.

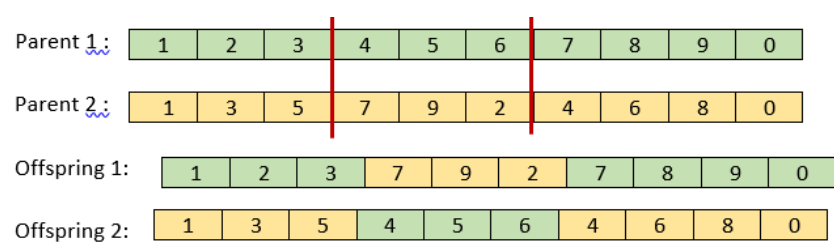

Figure 7. K-point Crossover

2.4.3 Shuffle Crossover: In this method two chromosomes are selected in which genes are shuffled randomly but in the same way. It uses 1point crossover technique by randomly selecting a point for crossover and then combines both the chromosome to create new offspring. After crossover again the genes in offspring are unshuffled in same way as they have been shuffled.[7]

2.4.4 Reduced Surrogate Crossover: This technique minimizes the crossover operation in case if both the parents having same genes. In these technique, first it check for the individual gene in the parents. It creates list of crossover points where the gene of the both parents are different. Suppose if there is no change in the gene of parents then no action is taken. Suppose if the parents are differing in more than 1 genes then all the points are recorded . so that it can choose any one point from the list and perform 1point crossover operation to create the new offspring.[7]

2.4.5 Uniform crossover: Uniform crossover provides uniformity in combining the genes of both parents. In this technique a coin is tossed to decide whether the chromosome to be included in the offspring. Assigns each gene in the new offspring is created by copying the bit from the parent based on the corresponding bit in the binary crossover mask of same length as the length of both parents[c-2] . if the bit in the binary crossover mask is 1 , then the resultant bit in the offspring is copied from the first parent else it copied from the second parent. 
2.4.6 Average Crossover: This technology is based on the value based crossover technique. In this technique only one offspring is generated from the parents. Offspring is created by taking the average of the corresponding gene of the two parents. For Example

Parent 1 : 34526789

Parent 2 : 45862467

Offspring 1: 34644578

2.4.7 Discrete Crossover: In Discrete Crossover only one child is generated from two parents. Gene in the offspring is selected from the parents uniformly. The random real number decides from which parent to take the genes for child. For Example

Parent 1: 111010010

Parent 2: 100010110

Offspring 1: 110010110

\subsection{Mutation}

Mutation is carried out after crossover phase. In this phase, it allows to have small random tweak in the chromosome in order to get a new solution. This is used to maintain the diversity within the population and prevent premature convergence. Many types of mutation operator are available like mirror mutation and binary-flipping mutation, directed mutation, covariance matrix adaption evolution strategy, nonuniform mutation, adaptive mutation etc., [6]

Finally the algorithm terminates if the population has converged. Then it is said to have a set of solutions to the problem.

\subsection{Termination}

Finally the genetic algorithm terminates when it identifies any of the following conditions are met[9].

*When the solution satisfies the minimum criteria specified

*When a specified number of iteration are performed

* When a definite amount of computation time or resource is utilized

* When it reaches a certain pre-defined value

* When there is no change in the value for a sequence of several consecutive generations

\section{Pseudo code for Genetic Algorithm}

Pseudo code for genetic algorithms is given below[10]

TABLE 2: PSEUDO CODE FOR GENETIC ALGORITHM

START
GENERATE RANDOM POPULATION
WHILE NOT
COMPUTE FITNESS FUNCTION
SELECTION OF PARENTS
APPL YING CROSSOVER OPERATION
MUTATION OPERATION
UNTIL THE BEST SOLUTION IS RETURNED
STOP

\section{CONCLUSION}

The successful implementation of GAs mainly depends on the selection of parents for generating new offspring, efficient crossover operator and mutation search operators to guide the system to obtain optimal solution for the given problem. This paper discuss about the working of Genetic algorithm in step by step manner. It discusses the seven wellknown selection method of parents for GA and standard crossover operators present in the GA. Each selection method and crossover operator has its own merits and demerits. Based on the type of application, suitable selection method and crossover method can be applied.

\section{REFERENCES}

[1]. Mansi Gangwar, Maiya Din,. K. Jha, "Comparative Study Of Selection Methods In Genetic Algorithm", International Journal of Soft Computing and Artificial Intelligence, Volume-5, Issue-1, May-2017, Pp-37-40.

[2]. Khalid Jebari, Mohammed Madiafi , "Selection Methods for Genetic Algorithms", International Journal of Emerging Science, 
Volume-3, Issue-4, December 2013, Pp:333344

[3]. Nidhi,"A Comparative Analysis of Genetic Algorithm Selection Techniques", International Research Journal of Engineering and Technology (IRJET), Volume: 04 Issue: 07 , July -2017, Pp: 2453-2455.

[4]. Nisha Saini, "Review of Selection Methods in Genetic Algorithms", International Journal Of Engineering And Computer Science, Volume 6 Issue 12 December 2017, Page No. 2226122263

[5]. Saneh Lata Yadav, Asha Sohal, "Study of the various selection techniques in Genetic Algorithms" , International Journal of Engineering, Science and Mathematics, Vol. 6 Issue 3, July 2017, Pp:198-204

[6]. Siew Mooi Lim, et.al., "Crossover and Mutation Operators of Genetic Algorithms", International Journal of Machine Learning and Computing, Vol. 7, No. 1, February 2017, Pp:9-12.

[7]. ABDOUN Otman, ABOUCHABAKA Jaafar, "A Comparative Study of Adaptive Crossover Operators for Genetic Algorithms to Resolve the Traveling Salesman Problem", International Journal of Computer Applications ,Volume 31- No.11, October 2011, Pp: 0975 - 8887.

[8]. A.J. Umbarkar and P.D. Sheth, "CROSSOVER OPERATORS IN GENETIC ALGORITHMS: A REVIEW", ICTACT JOURNAL ON SOFT COMPUTING, OCTOBER 2015, VOLUME: 06, ISSUE: 01, Pp:1083-1092

[9]. Saroj Kaushik, Sunita Tiwari, “Soft Computing Fundamentals, Techniques and Applications", Mc Graw Hill Education.

[10]. https://towardsdatascience.com/introductionto-genetic-algorithms-including-examplecode-e396e98d8bf3

\section{Cite this article as :}

Dr. K. Santhi, Dr. V. Vinodhini, "Study on Selection Methods of Parents and Crossover in Genetic Algorithm", International Journal of Scientific Research in Computer Science, Engineering and Information Technology (IJSRCSEIT), ISSN : 24563307, Volume 7, Issue 2, pp.280-285, March-April2021. Available at doi : https://doi.org/10.32628/CSEIT217256 Journal URL : https://ijsrcseit.com/CSEIT217256 